\title{
Supporting Information: Photocatalytic Water Splitting Reaction Catalyzed by Ion-exchanged Salts of Potassium Poly (heptazine imide) 2D Materials
}

Sudhir K. Sahoo, ${ }^{1}$ Ivo F. Teixeira, ${ }^{2,3}$ Aakash Naik, ${ }^{1}$ Julian Heske, ${ }^{1,2}$ Daniel Cruz, ${ }^{4,5}$ Markus Antonietti, ${ }^{2}$ Aleksandr Savateev, ${ }^{2}$ and Thomas D. Kühne ${ }^{1,6, *}$

${ }^{1}$ Dynamics of Condensed Mater and Center for Sustainable System Design, Chair of Theoretical Chemistry, University of Paderborn, Warburger Str. 100, D-33098 Paderborn, Germany

${ }^{2}$ Department of Colloid Chemistry, Max Planck Institute of Colloids and Interfaces, Am Mühlenberg 1, D-14476 Potsdam, Germany

${ }^{3}$ Department of Chemistry, Federal University of São Carlo, 13565-905, São Carlos, SP, Brazil.

${ }^{4}$ Fritz-Haber-Institut der Max-Planck-Gesellschaft, Faradayweg 4-6, 14195-Berlin, Germany

${ }^{5}$ Department of Heterogeneous Reactions, Max Planck Institute for Chemical Energy Conversion, Mühleim an der Ruhr, Germany

${ }^{6}$ Paderborn Center for Parallel Computing and Institute for Lightweight Design, University of Paderborn, Warburger Str. 100, D-33098 Paderborn, Germany

Email: tdkuehne@mail.uni-paderborn.de 

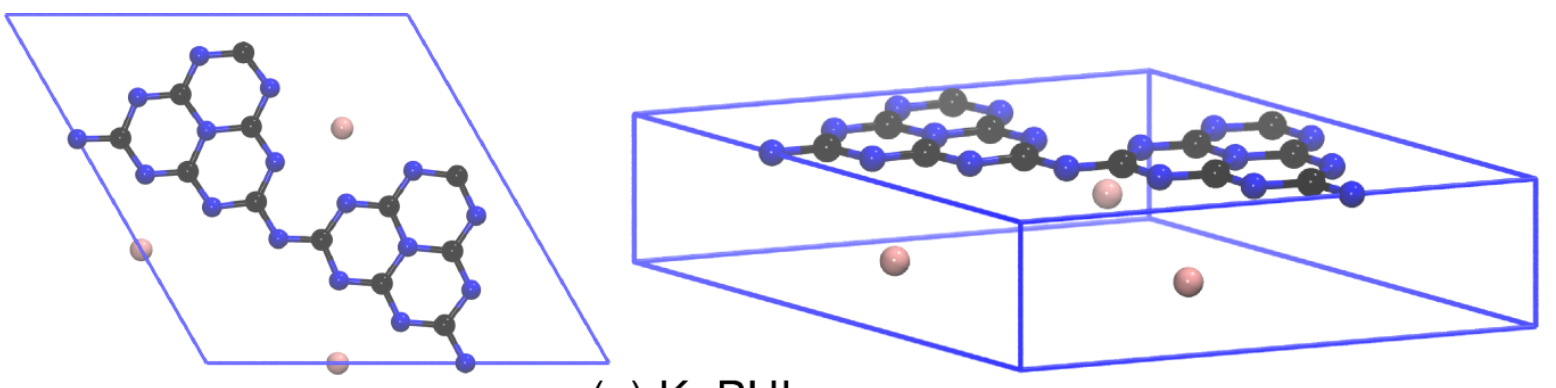

(a) $\mathrm{K}-\mathrm{PHI}$
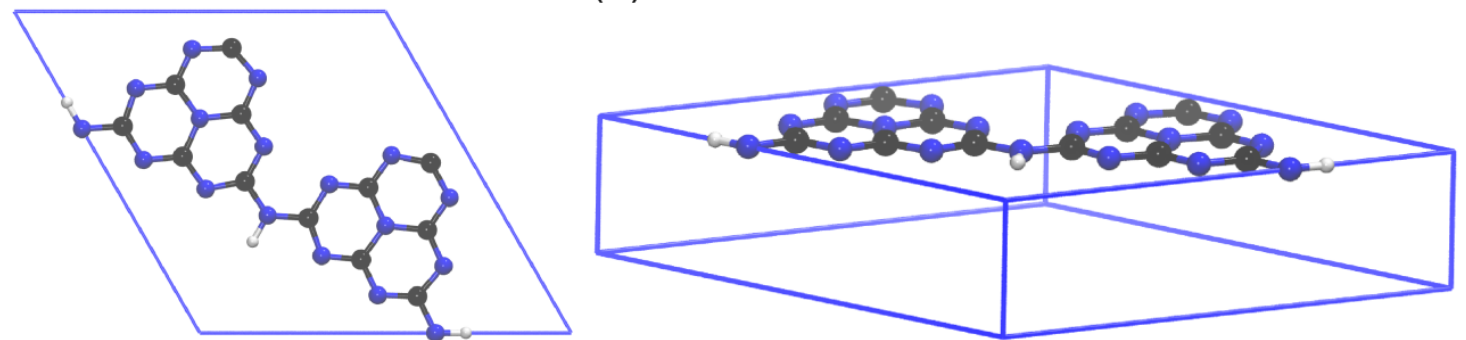

(b) $\mathrm{H}-\mathrm{PHI}$
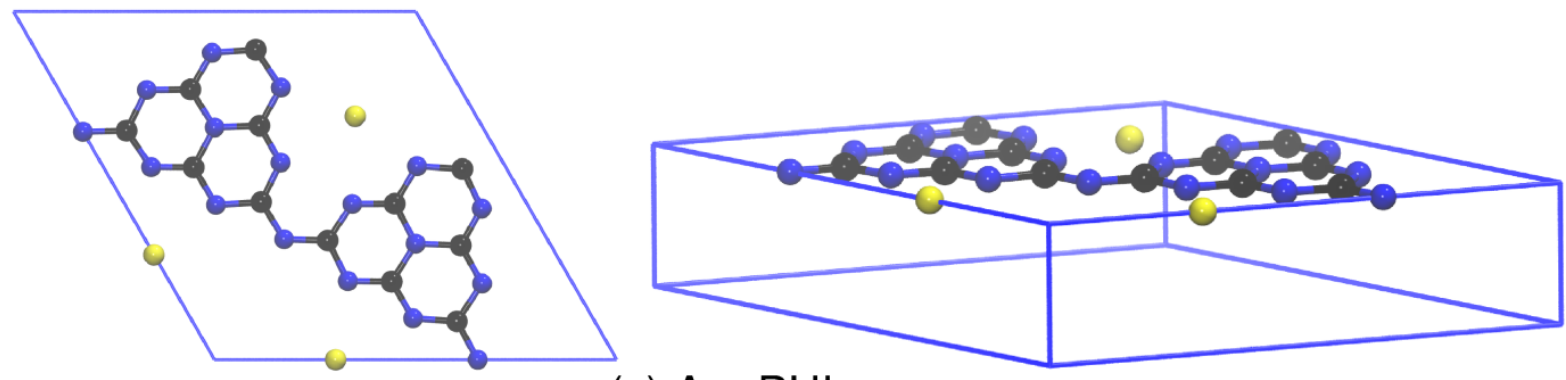

(c) Au-PHI
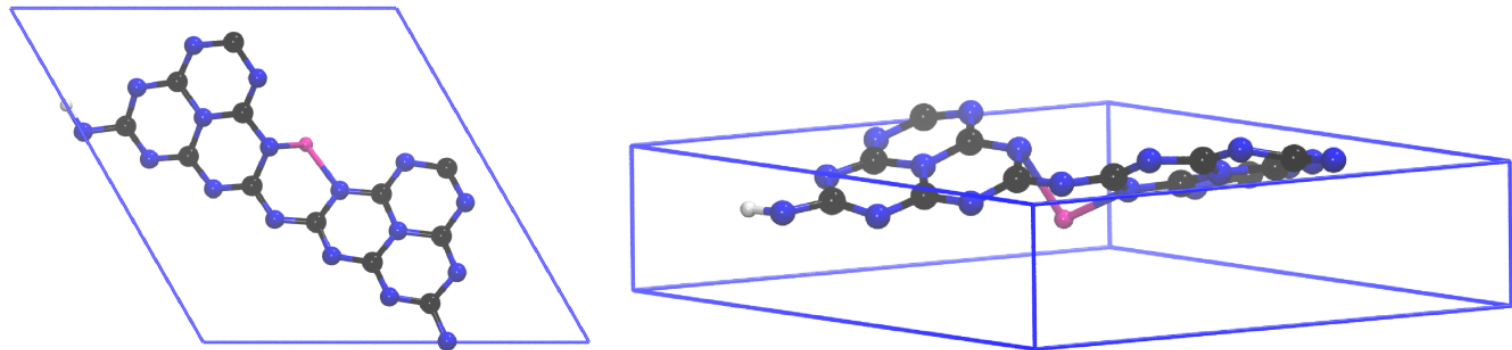

(d) $\mathrm{Mg}-\mathrm{PHI}$
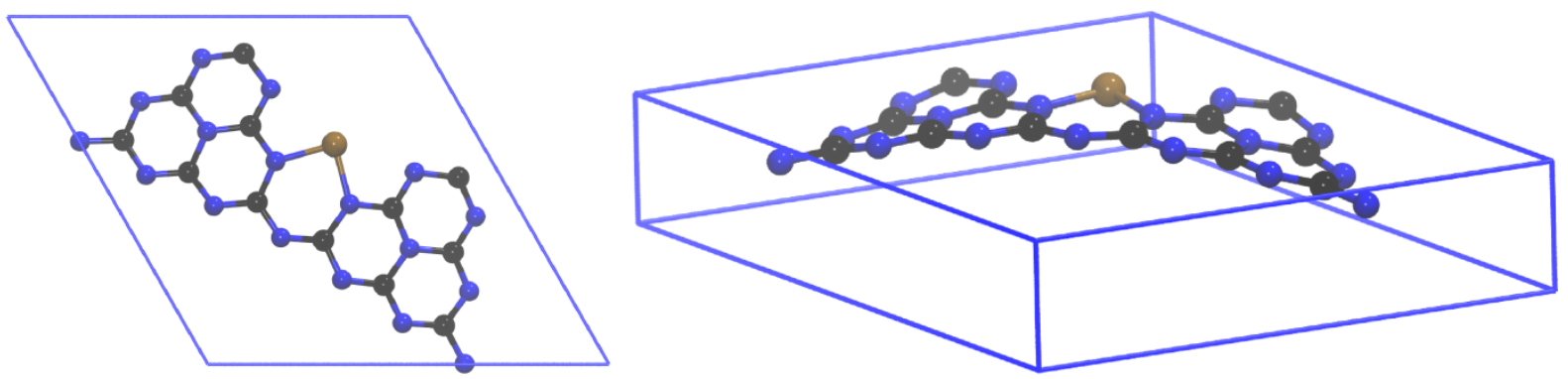

(e) Ru-PHI

Figure S1: The top-view (left side) and side view (right side) of the bulk structures of M-PHI obtained from our DFT calculations. Atoms color: C-black, N-blue, H-white, K-pink, Auyellow, Mg-magenta and Ru-ochre. 

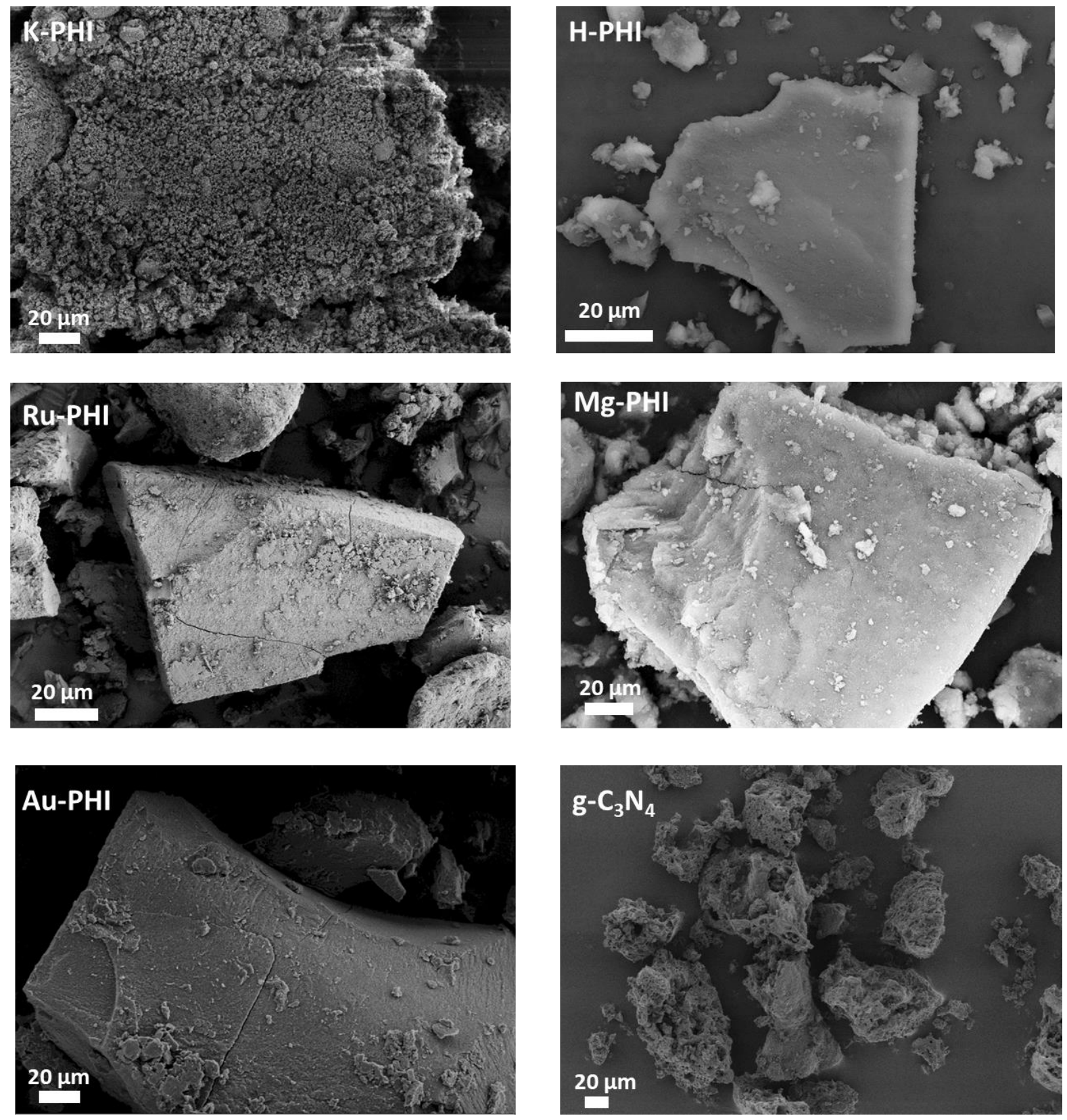

Figure S2: Scanning electron microscopy (SEM) characterization of the K-PHI, metal exchanged PHI samples and $\mathrm{g}-\mathrm{C}_{3} \mathrm{~N}_{4}$. The samples display similar morphologies, and the metal exchanged samples seem to be bulkier. 
Table S1: Elemental composition of long-layered M-PHI according to EDS and elemental analysis.

\begin{tabular}{|c|c|c|c|c|c|c|c|}
\hline Sample & $\mathrm{C}(\%)$ & $\mathrm{N}(\%)$ & $\mathrm{H}(\%)$ & $\mathrm{H}_{2} \mathrm{O}(\%)^{\mathrm{a}}$ & $\mathrm{K}(\%)$ & $\mathrm{M}(\%)$ & $\mathrm{C} / \mathrm{N}$ \\
\hline K-PHI & $26.5( \pm 2.0)$ & $40.7( \pm 2.0)$ & $1.96( \pm 0.7)$ & $11.7( \pm 1.5)$ & $14.9( \pm 2.13)$ & - & 0.65 \\
\hline H-PHI & $29.4( \pm 2.0)$ & $44.2( \pm 2.0)$ & $2.92( \pm 0.7)$ & $15.7( \pm 1.5)$ & $0.4( \pm 0.1)$ & - & 0.67 \\
\hline Mg-PHI & $25.9( \pm 2.0)$ & $40.5( \pm 2.0)$ & $2.48( \pm 0.7)$ & $11.2( \pm 1.5)$ & $3.3( \pm 0.28)$ & $2.0( \pm 0.1)$ & 0.64 \\
\hline Ru-PHI & $18.0( \pm 2.0)$ & $27.5( \pm 2.0)$ & $2.43( \pm 0.7)$ & $15.3( \pm 1.5)$ & $0.2( \pm 0.04)$ & $15.4( \pm 1.5)$ & 0.66 \\
\hline Au-PHI & $19.9( \pm 2.0)$ & $29.7( \pm 2.0)$ & $1.88( \pm 0.7)$ & $10.7( \pm 1.5)$ & $1.5( \pm 0.38)$ & $26.2( \pm 5.9)$ & 0.67 \\
\hline
\end{tabular}

${ }^{\text {a }}$ Calculated by the TGA weight loss.

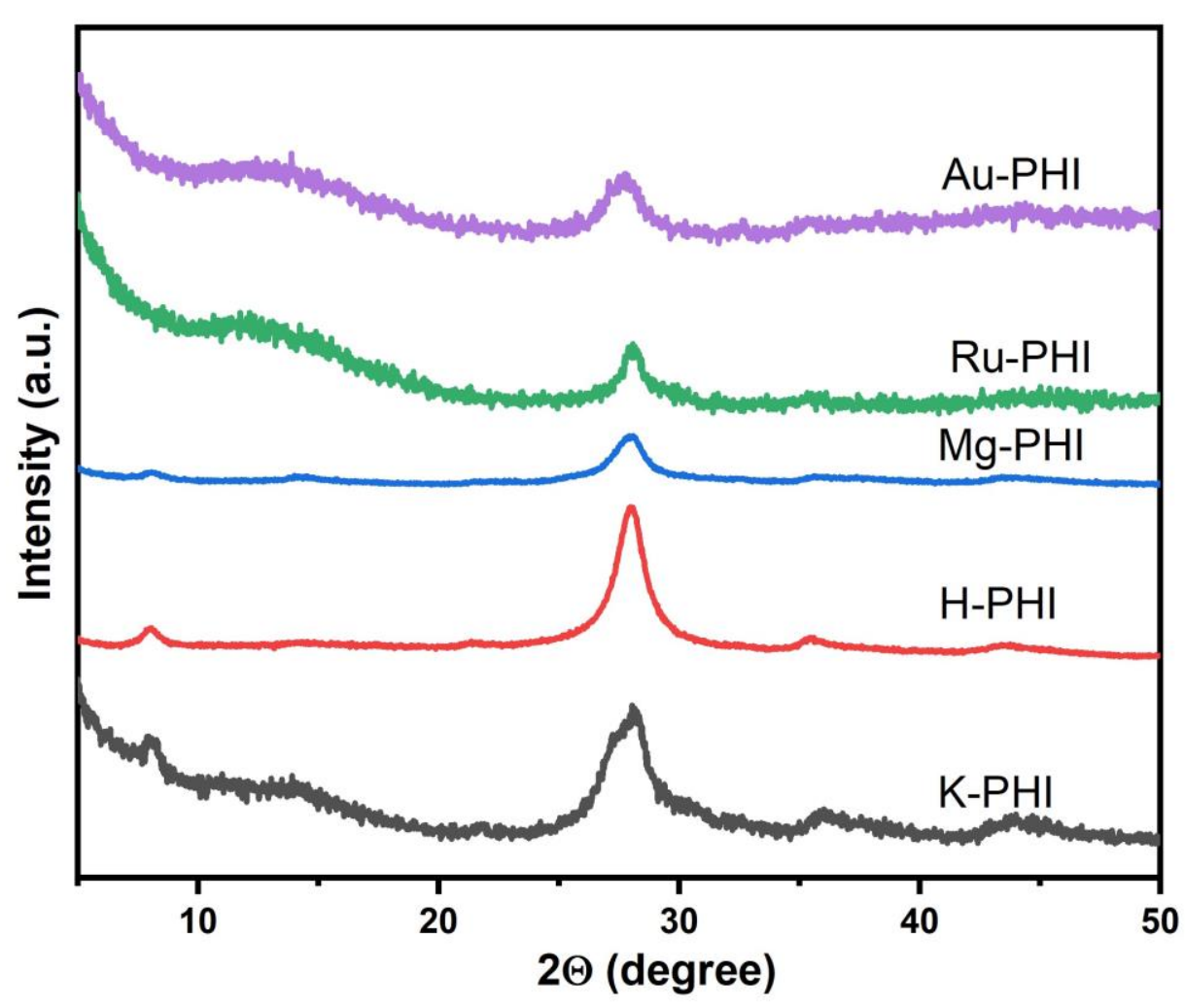

Figure S3: Powder X-ray diffraction patterns of M-PHI samples. 


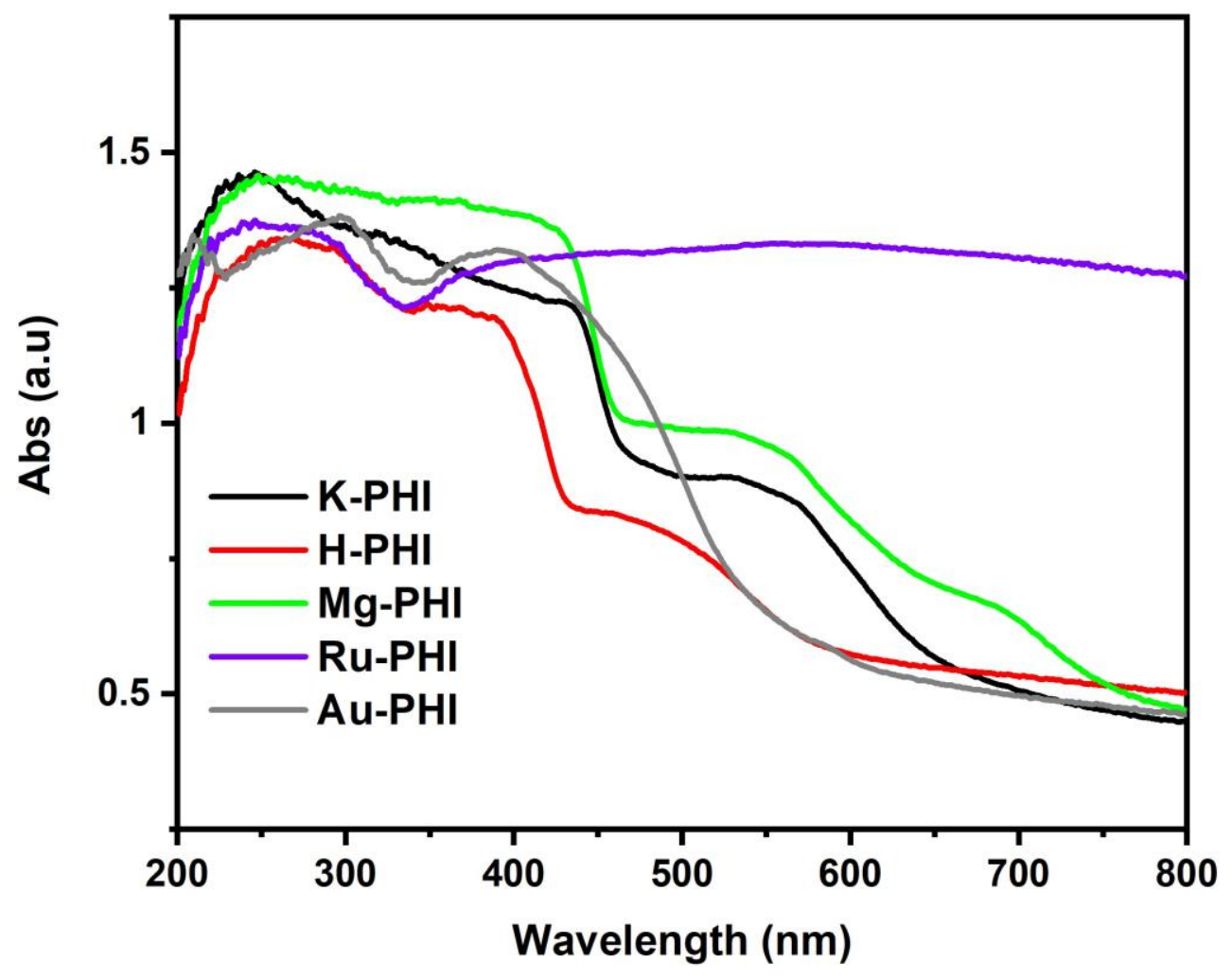

Figure S4: Steady-state UV-Visible absorption spectra for the M-PHI samples. 


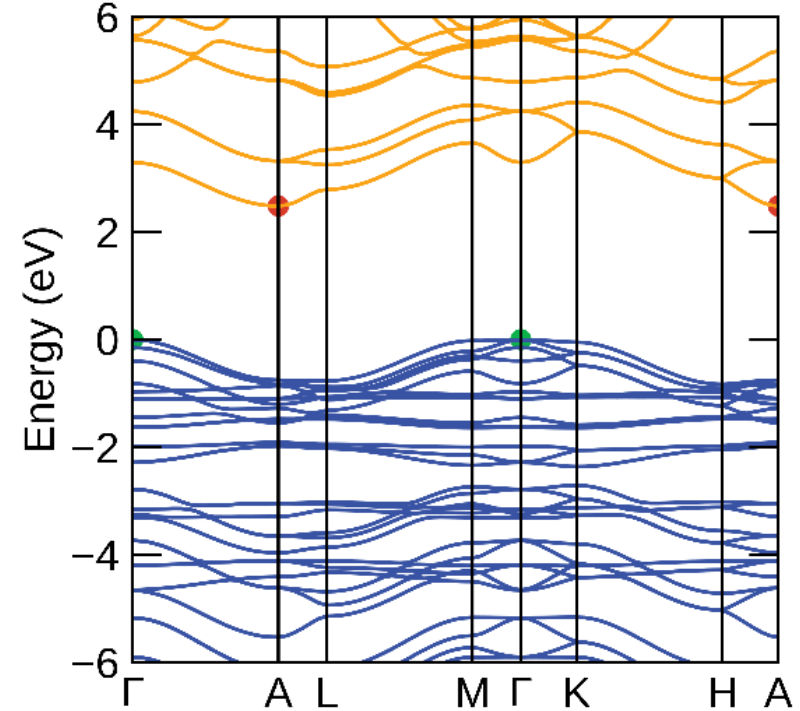

(a)

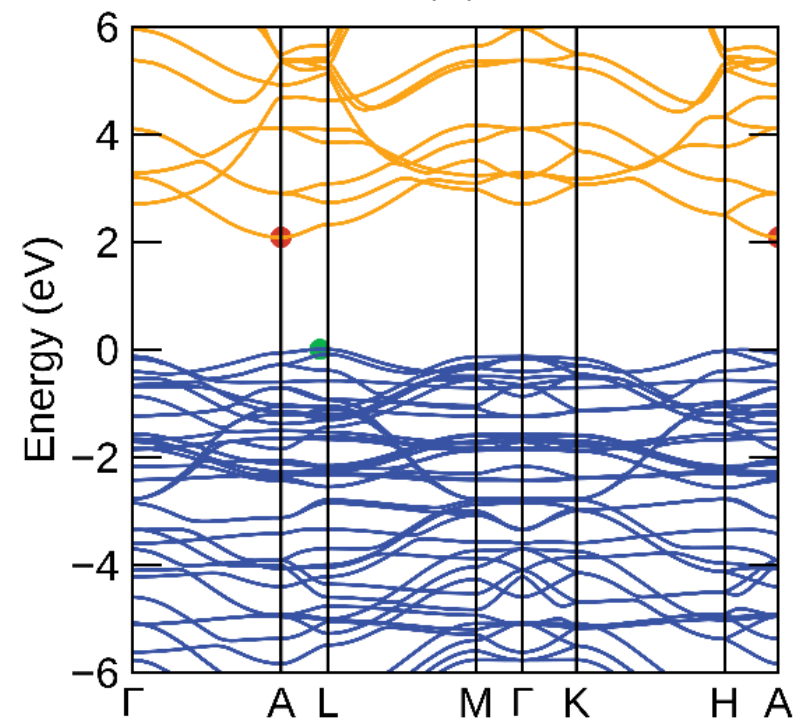

(c)

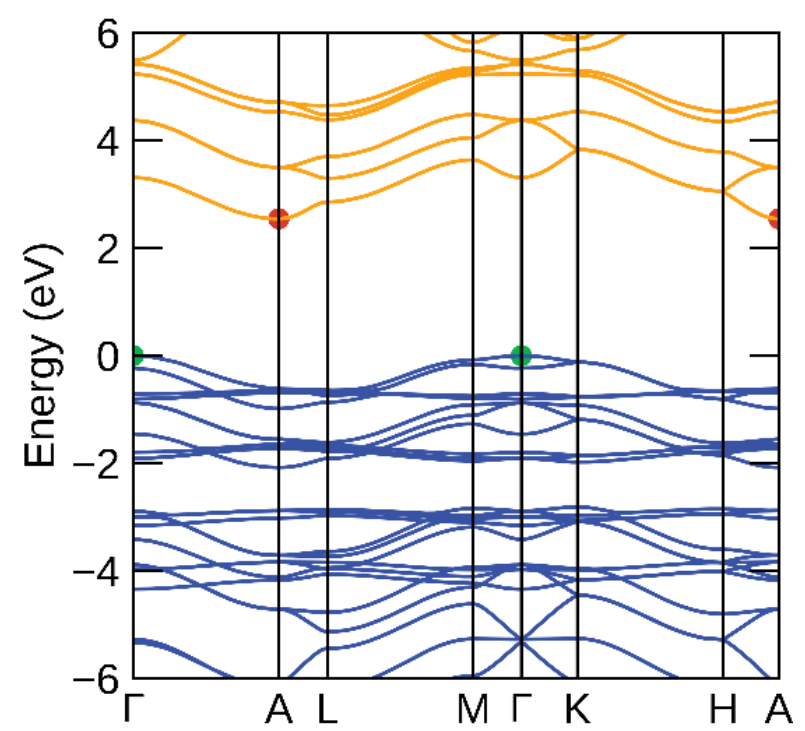

(b)

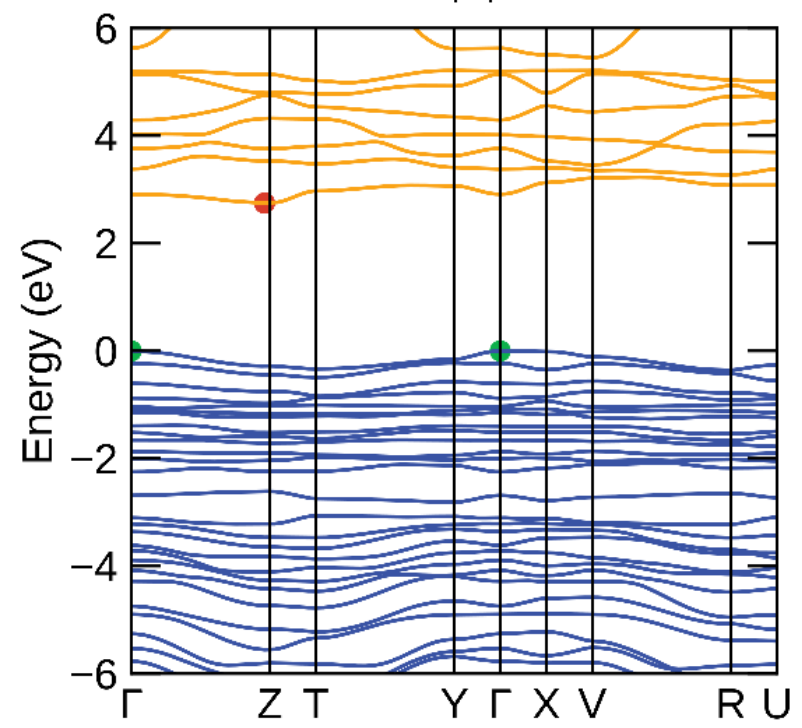

(d)

Figure S5: The electronic band structures of K-PHI (a), H-PHI (b), Au-PHI (c) and Mg-PHI (d) computed using hybrid HSE DFT functional. The blue and red color balls highlight the valence band maximum and conduction band minimum, respectively. 
Table S2: The effective electron $\left(m_{e}^{*}\right)$ and hole $\left(m_{h}^{*}\right)$ masses computed from band structures.

\begin{tabular}{|l|l|c|c|}
\hline Materials & & & Description \\
\hline K-PHI & $m_{e}^{*}$ & 1.666 & $A \rightarrow \Gamma$ \\
& $m_{e}^{*}$ & 0.585 & $A \rightarrow L$ \\
& $m_{h}^{*}$ & -1.373 & $\Gamma \rightarrow A$ \\
& $m_{h}^{*}$ & -6.894 & $\Gamma \rightarrow M$ \\
\hline H-PHI & $m_{e}^{*}$ & 2.763 & $A \rightarrow \Gamma$ \\
& $m_{e}^{*}$ & 0.583 & $A \rightarrow L$ \\
& $m_{h}^{*}$ & -1.327 & $\Gamma \rightarrow A$ \\
& $m_{h}^{*}$ & -1.770 & $\Gamma \rightarrow M$ \\
\hline Au-PHI & $m_{e}^{*}$ & 1.300 & $A \rightarrow \Gamma$ \\
& $m_{e}^{*}$ & 0.716 & $A \rightarrow L$ \\
& $m_{h}^{*}$ & -20.798 & Between $A-L \rightarrow L$ \\
& $m_{h}^{*}$ & -2.418 & Between $A-L \rightarrow A$ \\
\hline Mg-PHI & $m_{e}^{*}$ & 2.598 & Between $Z-\Gamma \rightarrow \Gamma$ \\
& $m_{h}^{*}$ & -1.728 & $\Gamma \rightarrow Z$ \\
& $m_{h}^{*}$ & -1.037 & $\Gamma \rightarrow Y$ \\
\hline
\end{tabular}

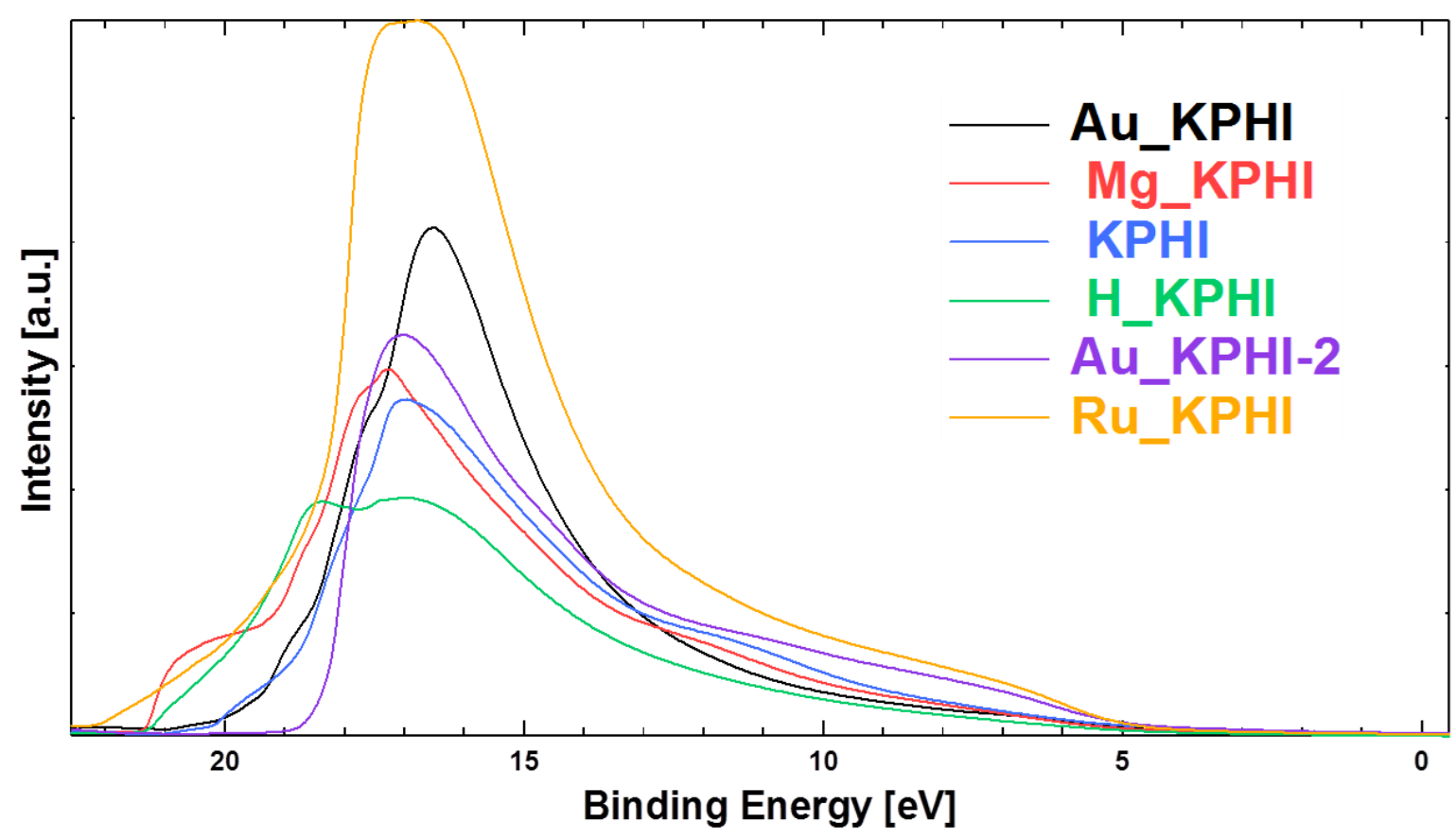

Figure S6: The ultraviolet photoelectron spectra of M-PHI samples used to calculate the valence band maximum. 


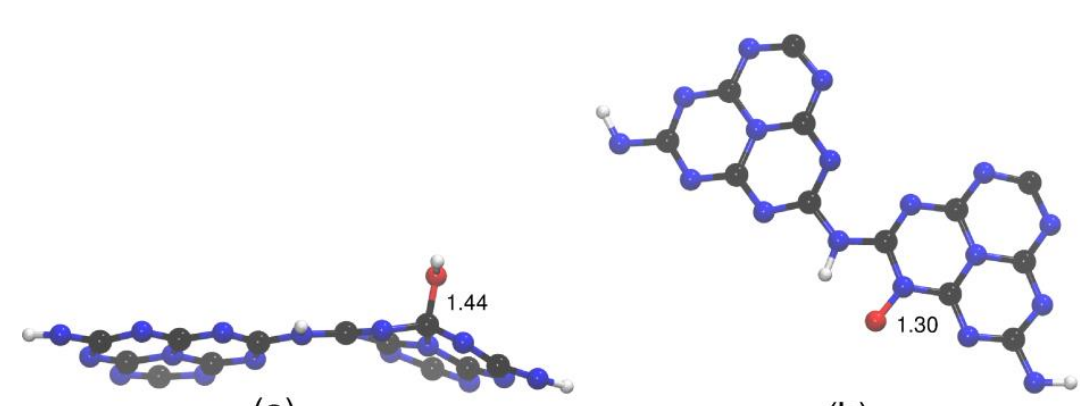

(a)

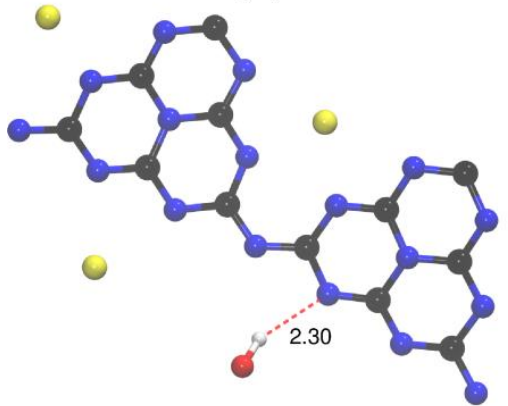

(d)

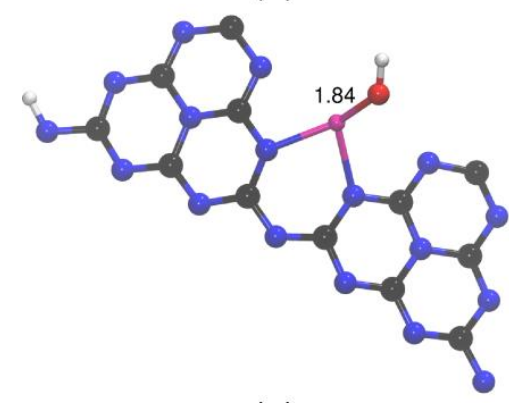

(g)

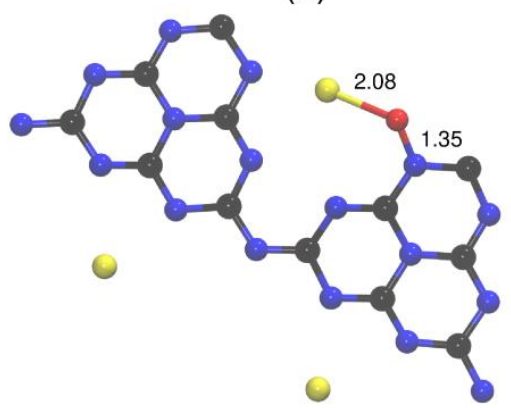

(e)

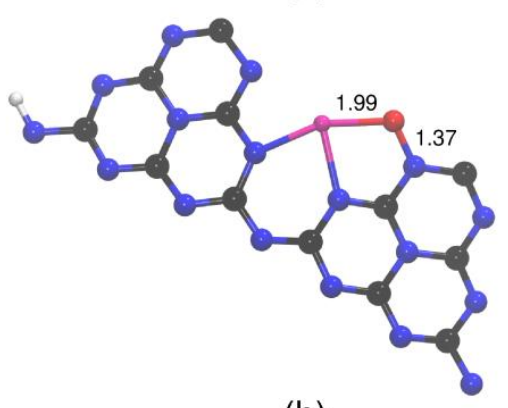

(h)

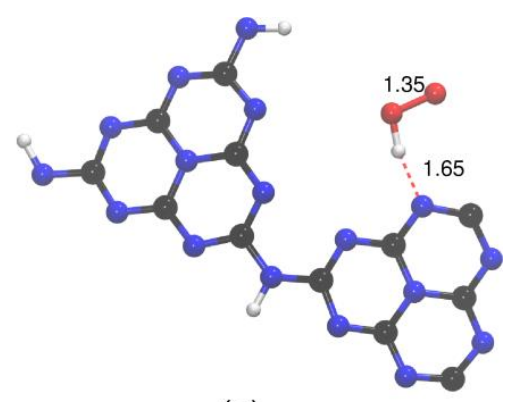

(c)

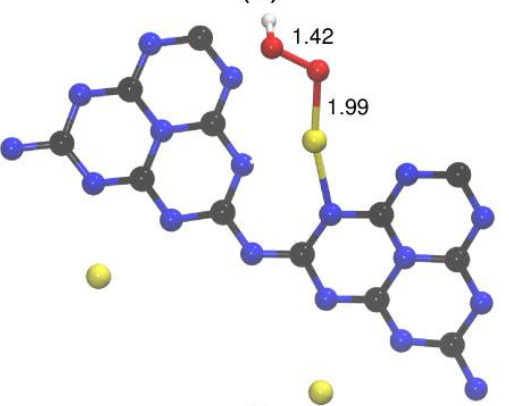

(f)

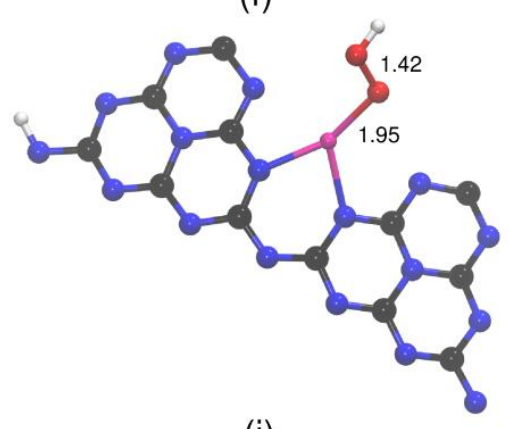

(i)

Figure S7: The optimized structures of intermediates $\mathrm{HO}^{*}(\mathrm{a}), \mathrm{O}^{*}$ (b) and $\mathrm{HOO}^{*}$ (c) on $\mathrm{H}-$ PHI; HO* (d), O* (e) and HOO* (f) on Au-PHI; HO* (g), O* (h) and HOO* (i) on Mg-PHI as photo-catalyst. Some of the selected bond distances (in $\AA$ unit) are marked. 


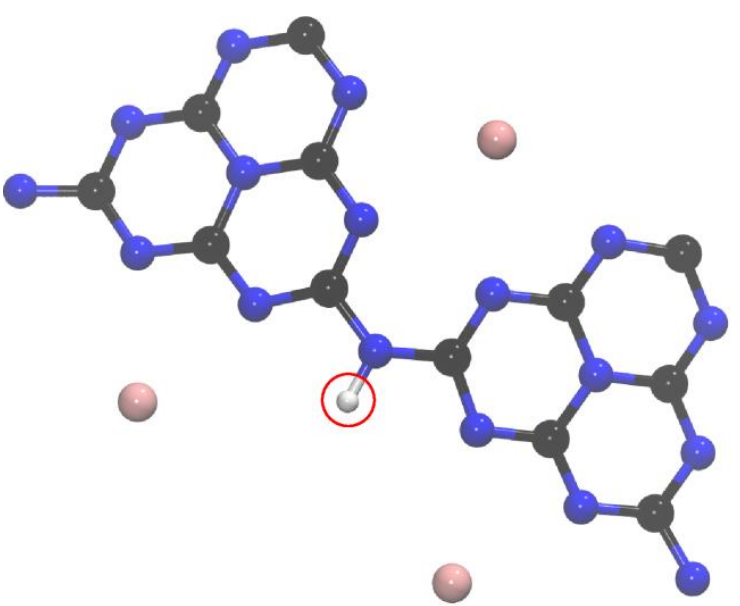

(a)

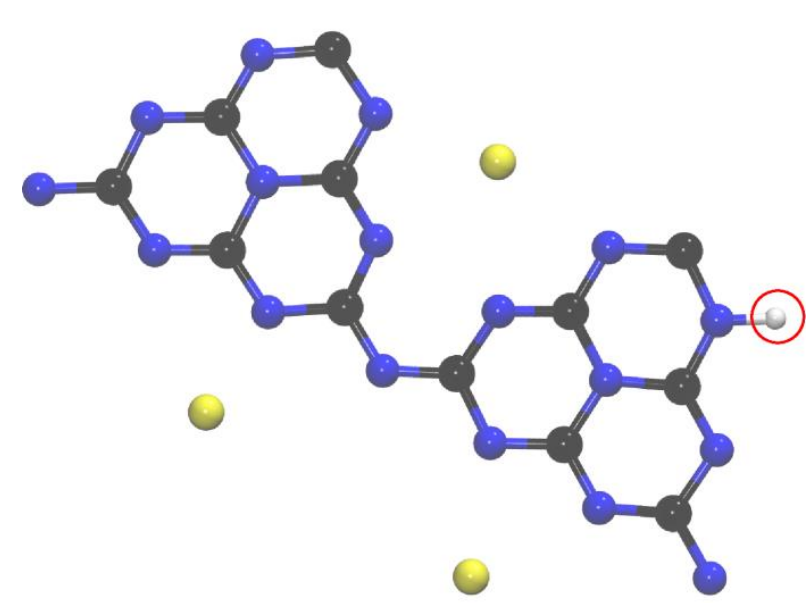

(c)

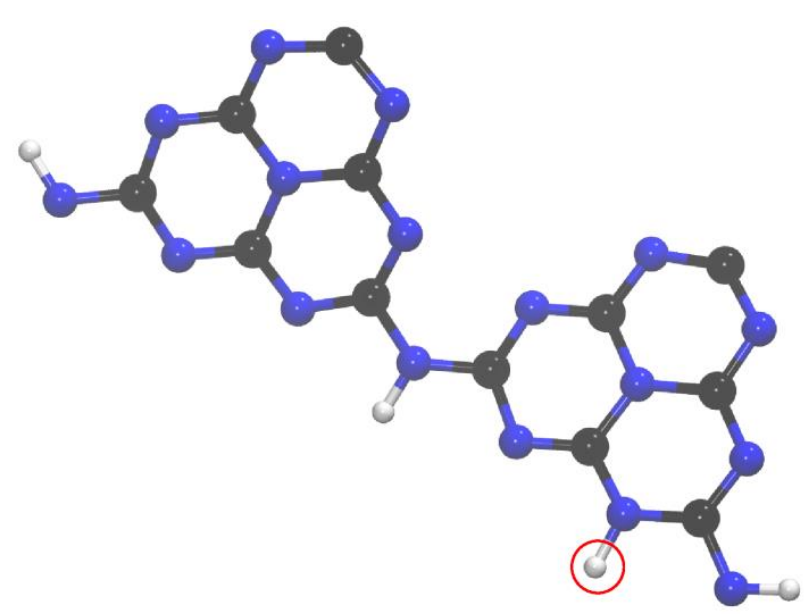

(b)

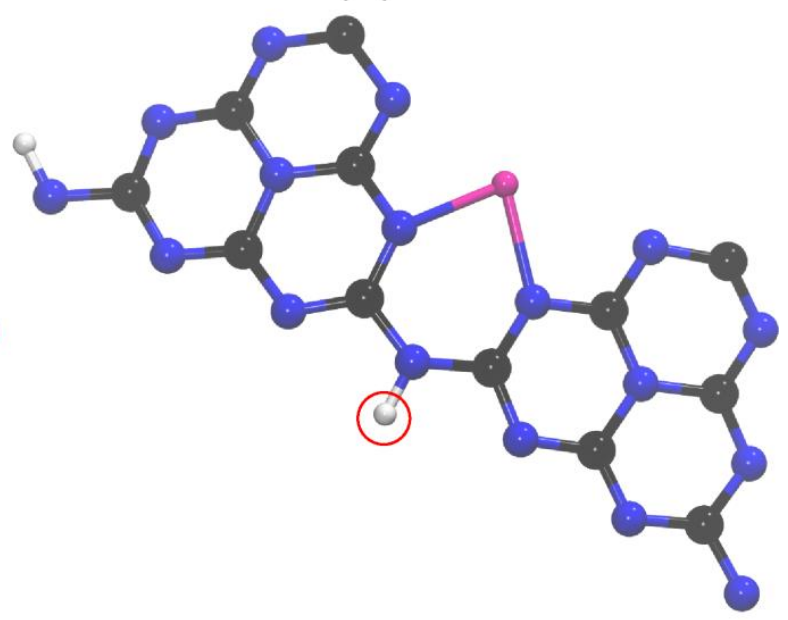

(d)

Figure S8: The optimized structure of $\mathrm{H}^{*}$ on K-PHI (a), H-PHI (b), Au-PHI (c) and Mg-PHI (d) obtained from the DFT calculations. The $\mathrm{H}$ atom is highlighted in the red color circle.

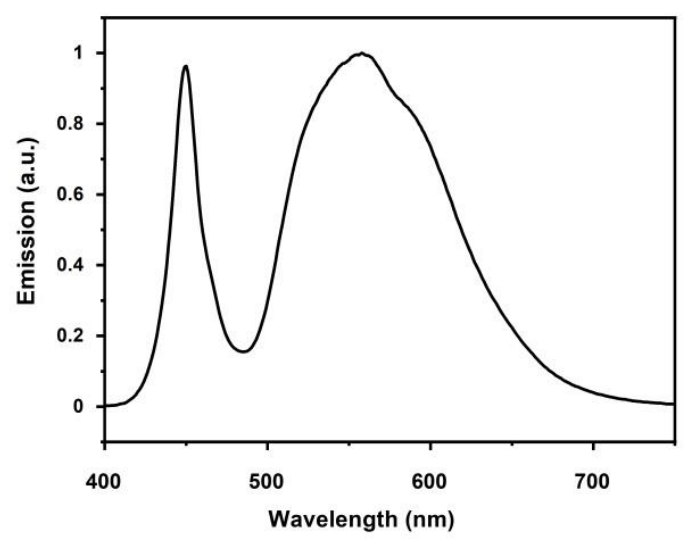

Figure S9: The spectrum of white LED used in the photocatalytic hydrogen evolution reaction. 